\title{
La responsabilité de la vie : l'autonomie dans la vulnérabilité
}

\section{Éric Pommier}

\section{(2) OpenEdition}

1 Journals

Édition électronique

URL : http://journals.openedition.org/alter/303

DOI : $10.4000 /$ alter.303

ISSN : 2558-7927

Éditeur :

Association ALTER, Archives Husserl (CNRS-UMR 8547)

\section{Édition imprimée}

Date de publication : 15 novembre 2014

Pagination : 163-179

ISBN : 978-2-9550449-0-2

ISSN : $1249-8947$

\section{Référence électronique}

Éric Pommier, «La responsabilité de la vie : l'autonomie dans la vulnérabilité », Alter [En ligne], 22 |

2014, mis en ligne le 01 décembre 2017, consulté le 10 décembre 2020. URL : http://

journals.openedition.org/alter/303; DOI : https://doi.org/10.4000/alter.303

Ce document a été généré automatiquement le 10 décembre 2020.

Revue Alter 


\title{
La responsabilité de la vie: l'autonomie dans la vulnérabilité
}

\author{
Éric Pommier
}

\section{NOTE DE L'AUTEUR}

Cet article a été rédigé dans le cadre du projet de post-doctorat Conicyt $n^{\circ} 3130574$.

1 Le regard jeté sur la vie souffre d'une certaine ambivalence au sein des analyses contemporaines, dans la mesure où celle-ci est tantôt présentée comme un bien fragile qu'il faut protéger et dont la prise en compte pourrait même conduire au renouvellement profond de notre culture ${ }^{2}$, tantôt, au contraire, comme une menace essentielle qui mettrait en question les fondements de la civilisation humaine ${ }^{3}$. D'un côté, on affirme que c'est à condition de reconnaître la vulnérabilité des vivants, y compris humains, que l'on sera susceptible de se reconnaître des devoirs envers eux. D'un autre côté, il s'agit de faire valoir le danger qu'il y a à réduire l'humanité, comprise dans son autonomie morale par différence avec l'animalité, au statut de simple espèce. Si c'est bien l'opposition entre la pensée de la vulnérabilité de la vie et celle de l'autonomie de l'homme qui structure le conflit que nous venons d'évoquer, l'on peut alors se demander si la mise en jeu de la philosophie de Hans Jonas ne permet pas de le surmonter au plan conceptuel et de présenter les linéaments d'une éthique qui, tout en s'adressant à la vie et en s'appuyant sur elle dans le but d'élargir le champ d'application de nos exigences morales, ne fasse pas pour autant l'économie du privilège que l'humanité occupe en son sein ${ }^{4}$. Dans un premier temps, nous ferons donc droit au positionnement de Hans Jonas au sein du panorama contemporain brièvement survolé en insistant sur les critiques explicites ou reconstruites que sa pensée encourt avant d'identifier ce qui nous semble être un préjugé commun à ces critiques. Il sera alors possible, dans un second moment, de mesurer la fécondité du dispositif jonassien au regard de ce préjugé qu'il surmonte. Dans un dernier moment, nous identifierons la source d'une fragilité de la pensée jonassienne de la vie et indiquerons dans quelle direction il nous semble envisageable de la résoudre. 


\section{Du conflit entre l'autonomie et la vulnérabilité}

On trouvera la trace d'une inquiétude devant la biologisation de l'existence humaine dans la critique que Karl-Otto Apel $^{5}$ adresse à Hans Jonas, promoteur d'un principe responsabilité, visant à préserver l'authenticité de la vie humaine face aux périls que les technologies lui font courir, soit de manière interne par le biais de l'ingénierie génétique, soit de manière externe par une modification d'ampleur de son habitat. En mettant au centre de l'éthique le simple souci de conserver l'espèce humaine vulnérable, en reléguant celui de la justice et de la dignité au second plan, l'auteur du Principe responsabilité rendrait impossible la pensée du progrès de l'histoire humaine et autoriserait donc des actes barbares. Dans un tel cadre, quelle norme interdirait par exemple le génocide d'une partie de l'humanité - sans doute ses représentants les plus défavorisés et vulnérables - si tel était le moyen requis pour préserver l'espèce humaine des dangers que la surpopulation pourrait lui faire courir? Quand bien même Apel reconnait que Jonas ne saurait soutenir un projet aussi inhumain, il n'en considère pas moins que l'abandon de la référence à l'idée de justice et de progrès dont le socle est l'autonomie humaine autorise une telle dérive.

Paradoxalement, cependant, la pensée de Jonas encourt ou pourrait encourir un type de reproche exactement contraire. C'est ainsi parce qu'il resterait fidèle au cadre de pensée humaniste centré sur l'autonomie, quoi qu'il en soit de son vœu de rompre avec l'anthropocentrisme, que sa philosophie serait impropre, d'une part à développer une véritable éthique médicale, d'autre part à se prolonger sous la forme d'une éthique environnementale. Paolo Becchi, par exemple ${ }^{6}$, fait remarquer que l'éthique médicale de Hans Jonas continue d'accorder au concept de personne d'obédience kantienne un rôle cardinal si bien que cette éthique a une valeur propre et est indépendante de l'éthique générale développée dans Le principe responsabilité qui prétend rompre, jusqu'à un certain point, avec l'éthique kantienne. On pourrait alors en tirer la conclusion que si la référence à l'autonomie continue de jouer un rôle majeur au sein de cette pensée, cette dernière ne permet pas de prendre en charge le respect dû au malade qui en est dépourvu à cause de la fragilité même de son existence. Et c'est seulement à condition de mettre l'accent sur sa vulnérabilité qu'il serait possible d'en faire un objet de considération authentique.

4 Symétriquement, en dépit même du désir jonassien d'intégrer la nature au sein du champ du respectable, il faudrait au contraire remarquer, d'une part, que s'il accorde une valeur aux individus vivants, il n'en accorde pas en revanche à la terre qui fait partie des écosystèmes, d'autre part, que c'est surtout l'homme qui se met en danger en mettant en danger la nature plutôt que la nature elle-même, comme si celle-ci n'était pas véritablement susceptible d'une catastrophe en soi $^{7}$. De ce point de vue, il faudrait bien plutôt se tourner du côté de l'éthique environnementale élaborée par exemple par John Baird Callicott afin de rompre définitivement avec le discours qui fait de l'autonomie (humaine) le centre de référence de toute valeur. C'est pourquoi lorsque Callicott pense, dans la continuité de Leopold, l'existence d'une valeur intrinsèque de la communauté biotique et appelle au devoir de la respecter, il marque une véritable rupture avec l'ensemble de la tradition philosophique que n'a pas su effectuer Hans Jonas.

5 Cependant, en dépit du caractère opposé de ces critiques réelles et possibles faites à l'encontre du mode de pensée jonassien, tantôt considéré comme étant trop centré sur la 
vie en son caractère de vulnérabilité (la vulnérabilité ne pouvant justifier à elle seule un principe de respect), tantôt au contraire comme étant trop proche de l'humanisme et par conséquent de l'autonomie (celle-ci ne permettant pas de penser le respect pour les écosystèmes et les vivants humains souffrants qui en sont dépourvus), il faut remarquer que celles-ci semblent se rejoindre dans la dépendance à un préjugé commun, selon lequel l'humanité est toujours l'autre de la vie, l'autonomie l'autre de la vulnérabilité. Ceci parait évident dans le cas de la philosophie de Apel, pour laquelle l'humanité n'a de valeur qu'en référence à la mise en œuvre d'un principe d'argumentation qui est indépendant de toute référence à la vie ou à notre incarnation. La vie n'a pas de valeur en soi et mon humanité ne tire pas sa valeur de son inscription dans une nature. Si Apel s'éloigne de Kant parce qu'il considère que le règne des fins est un reste de métaphysique qui doit être dépassé, il le rejoint cependant dans son affirmation d'une valeur de l'humanité dont la dignité est irréductible à la vie, la fragilité de cette dernière ne pouvant constituer une valeur suscitant le respect. La nature reste un ensemble parfaitement objectivable axiologiquement neutre tandis que l'humanité est la seule source, par soi-même, de valeur.

6 Mais ce constat théorique nous semble valoir également, et très paradoxalement, pour les tenants d'une éthique médicale qui consacrent la valeur de vulnérabilité au détriment de l'autonomie ou les partisans d'une éthique environnementale telle que celle de Callicott qui font porter l'accent sur la valeur intrinsèque de la communauté biotique. Dans le premier cas, c'est la vulnérabilité qui est source de valeur sans qu'il soit besoin de préserver un horizon d'autonomie à cet effet. Mais on voit mal en ce cas comment elle peut seule conférer à la vie une valeur digne d'être vécue et comment le médecin, qui voudrait la préserver à ce seul titre, au nom même de l'impératif de sollicitude, ne courrait pas le risque d'infliger à celui qui est d'une vulnérabilité extrême, à la limite de la mort par exemple, un destin cruel. C'est donc bien qu'implicitement le médecin, qui soigne la vie diminuée, le fait en vertu d'un principe d'humanité qui conserve une relation avec la valeur d'autonomie, actuelle ou possible. Faute de penser le lien originaire entre la vulnérabilité et l'autonomie, cette dernière est donc clandestinement réintégrée lorsqu'il s'agit de soigner la vie vulnérable.

Dans le second cas, celui de l'éthique de Callicott, il nous semble également que le partage strict entre l'humanité et la nature, entre l'autonomie de l'homme et la vulnérabilité de la nature est maintenu en un dualisme peut-être encore plus strict que dans le cas de figure précédent ${ }^{8}$.

8 (1) De fait, si Callicott accorde une valeur intrinsèque à la communauté biotique, il n'en reste pas moins que seul l'homme est susceptible de lui concéder cette valeur. C'est l'homme qui valorise ces ensembles pour leur accorder une valeur indépendante de tout usage. Il peut certes indiquer des raisons d'une telle valorisation mais Callicott semble refuser l'idée d'une valeur qui serait interne à la nature, inscrite en elle, qui lui appartiendrait de manière véritablement intrinsèque, indépendamment donc de l'existence de l'homme. Le lieu de la valeur est peut-être la nature mais sa source en est l'homme. Callicott maintient donc fermement le cadre de la pensée moderne de l'opposition du sujet et de l'objet tel que l'a établi Descartes ${ }^{9}$ (ce que démentirait pourtant une description du phénomène de la vie). Seul un sujet humain peut valoriser pour luimême, même s'il peut valoriser selon Callicott des ensembles non humains, non subjectifs comme les ensembles éco-systémiques. 
9 (2) On pourrait ajouter que ce pouvoir de valoriser propre à l'homme semble l'arracher au règne de la nature, puisque ce n'est pas parce qu'il en est ou qu'il en provient qu'il peut la valoriser et se reconnaître des devoirs envers elle (contrairement à ce qui se passe dans la pensée de Jonas, qui redescend de la valeur que l'homme s'attribue en tant que vivant à celle des vivants en général, grâce à un anthropomorphisme positif que nous évoquerons plus loin), mais seulement parce qu'il est humain. Certes Callicott ne manque pas de s'inspirer de la genèse darwinienne de la morale (que nous ne pouvons analyser ici) ${ }^{10}$ pour indiquer comment peut naturellement apparaître chez l'homme un sens du devoir à l'égard de la nature, qui elle-même ne peut donc plus être conçue de manière axiologiquement neutre ${ }^{11}$. Cependant faute de proposer une ontologie qui permette de rendre compte de l'émergence de la valeur comme telle, c'est-à-dire de la capacité du vivant à poser ce qui n'est pas comme ayant à être, ce qui a par exemple pour effet d'inhiber la violence égoïste au profit de la « reconnaissance implicite » d'autrui et de ses "droits propres », Callicott reste dépendant d'une conception objectivante de la nature, qui explique l'origine de la valeur à partir de l'exigence reproductive de l'espèce comprise de manière mécanique. Ainsi même si d'un point de vue «intérieur », c'est-à-dire du point de vue du vivant lui-même, on peut comprendre selon Callicott son comportement eu égard au respect d'une valeur intrinsèque, qui prend donc en considération l'autre individu vivant pour lui-même, il n'en reste pas moins que l'accès à ce point de vue « intérieur » n'est pas justifié pour lui-même et qu'il semble donc renvoyé, au bout du compte, au statut d'apparence sans valeur de vérité, cette dernière étant délivrée par le jugement de la sociobiologie scientifique ${ }^{12}$. On peut certes constater un altruisme intraspécifique mais ce phénomène n'est pas tant décrit pour lui-même selon son mode d'être propre que réduit à un mécanisme objectif qui fait de la nécessité reproductive, entendue comme mécanisme de survie de l'espèce, non pas tant la raison justifiant un «comportement» ou une "conduite» animale que la cause d'un automatisme qui se perpétue ${ }^{13}$.

10 (3) Enfin, pour échapper à l'objection de fascisme écologique en vertu de laquelle des mesures attentatoires à la dignité humaine pourraient être prises au nom du principe de conservation de la vie et de la nature et sans céder sur la nécessité de se reconnaître des devoirs écologiques, Callicott fait valoir, d'une part, que l'éthique de la terre s'ajoute ${ }^{14}$ mais ne se substitue pas aux droits de l'homme, puisqu'il y a une hiérarchie descendante des devoirs depuis les plus anciens et les plus personnels (qui sont donc plus importants) jusqu'aux plus récents et plus impersonnels, d'autre part « que les enjeux les plus forts [... ] génèrent des devoirs qui l'emportent sur les devoirs générés par des enjeux plus faibles $»^{15}$. En vertu de ce premier principe, un devoir envers l'homme aura donc plus d'importance qu'un devoir envers la nature. Cependant, en vertu du second, ce devoir envers l'homme peut être modifié afin de prendre en compte un devoir envers la nature. Mieux vaut par exemple protéger la vie économique de bûcherons que celle d'une forêt sauf s'il n'y a pas de commune mesure entre la destruction d'une forêt et des espèces qu'elle abrite et la délocalisation de bûcherons ${ }^{16}$. Cependant, en dépit de la force de cette analyse, il apparaît que le premier principe de hiérarchisation des devoirs qui met l'humanité au-dessus de la nature n'est pas justifié autrement que de manière factuelle en affirmant que le sentiment d'appartenance à celle-là est plus ancien et personnel que le sentiment d'appartenance à celle-ci, si bien que le primat de l'humanité est posé sans plus de justification axiologique. En outre, le second principe qui permet la prise en compte de devoirs écologiques semble relever d'un certain arbitraire, puisqu'il apparaît difficile de 
savoir ce qu'est au juste un enjeu plus fort ou plus faible, autrement qu'en faisant appel à un certain subjectivisme de l'opinion.

11 Ainsi Callicott semble acter une hiérarchie sans la justifier au plan des principes, sans expliquer pourquoi l'homme a plus de valeur en soi que le reste de la nature, sans indiquer véritablement l'origine de cette valeur tandis que la reconnaissance des devoirs écologiques est renvoyée à l'appréciation subjective d'un devoir qui dans certaines situations sera fort. On a alors l'impression non seulement que l'homme est la valeur absolue de même que l'origine de toute valeur mais également, et encore une fois, que cette valeur possédée était indépendante de son inscription naturelle au point de lui venir d'ailleurs. Tout se passe alors comme si, en dépit du vœu de penser le caractère intrinsèque de la valeur naturelle, Callicott était renvoyé à un dualisme de l'homme (dont l'autonomie lui permettait de s'assigner une valeur) et de la nature (dont la vulnérabilité motivait un respect qui revenait en dernier ressort à l'homme, selon la modalité de l'obligation indirecte de type kantien).

On en verrait, me semble-t-il, la raison dans sa dépendance ininterrogée à l'ontologie de la science de laquelle il hérite une représentation objectivante de la nature qui lui interdit véritablement de penser la valeur dans la nature et qui le contraint à en identifier le siège dans l'homme seul, par différence avec ce règne objectif du naturel. En cela, donc, Callicott reconduirait un humanisme qu'il semble avoir voulu contester ou élargir. C'est d'ailleurs ce qu'on peut lire dans l'appréciation que Catherine Larrère fait, au bout du compte, de l'éthique de Callicott dans Les philosophies de l'environnement ${ }^{17}$. D'une part, en effet, il est incontestable, comme l'écrit Catherine Larrère à propos de Callicott, que « la situation éthique qui place les êtres humains dans la communauté biotique procède d'une insertion ontologique", si bien qu' «attribuer une valeur à une entité ce n'est pas lui conférer, de l'extérieur, une caractéristique qui lui serait étrangère, c'est actualiser une relation qui existe déjà, c'est spécifier l'appartenance de l'homme à la communauté biotique », de sorte que la valeur doit ontologiquement être comprise comme l'expression d'une relation d'appartenance positive de l'homme à son environnement. D'autre part, cependant, « le tort de Callicott est de pousser jusqu'au bout l'analogie avec la modernité, de chercher, pour ce qui la suit, une unité de même type. Sans doute la synthèse moderne est-elle impressionnante, mais c'est une synthèse réductionniste, qui fait de la mécanique la raison d'être de tous les autres domaines ${ }^{18}$. Parce que Callicott conserverait malgré lui une représentation classique de la nature, il serait empêché de penser la nature selon cette catégorie relationnelle qui lui permettrait de loger la valeur au sein même de la nature et en remontant jusqu'à l'homme.

\section{L'éthique biocentrique de Hans Jonas}

13 Le maintien d'une opposition abstraite entre les notions d'autonomie humaine et de vulnérabilité naturelle reconduit les privilèges de l'humanisme classique si bien que c'est en contestant cette opposition qu'on pourra remettre en cause ce privilège et penser l'éthique selon son sens le plus propre et dans toute son extension. Or c'est précisément parce que Hans Jonas commence à surmonter un tel préjugé qu'il indique le sens dans lequel penser une éthique nouvelle.

En effet, dans Le phénomène de la vie ${ }^{19}$, Hans Jonas met en œuvre un anthropomorphisme positif, qui prend pour point de départ le témoignage que l'homme porte sur son propre corps afin d'en tirer des conclusions sur la nature même des êtres vivants. L'invalidation 
de l'anthropomorphisme traditionnel, c'est-à-dire de la projection de nos impressions subjectives sur des données objectives, au nom du postulat d'objectivité promu par la science moderne présuppose un découpage de la nature en une sphère objective et une sphère subjective que notre expérience même de la vie dément. Parce que nous faisons dans notre condition incarnée l'expérience d'un dépassement interne en vertu duquel le corps n'est jamais simplement expérimenté comme une chose étendue mais toujours aussi comme étant capable de sentiment et d'orientation et parce que nous ne pouvons jamais faire l'expérience d'un état psychique qui ne soit immédiatement aussi soumis à une forme d'extériorisation corporelle, nous sommes en mesure d'interpréter le sens même de la vie chez les autres vivants qui doivent également posséder, bien qu'à un autre degré, le type d'ouverture au monde dont nous faisons ainsi l'expérience. Le métabolisme qui manifeste ce pouvoir de la vie de se renouveler à travers ses échanges avec le milieu extérieur doit donc être compris comme ce premier surgissement de significations, qu'on ne peut pas référer à une conscience mais bien plutôt au mode d'être du vivant. De ce point de vue, il serait inadéquat de considérer qu'un monde puisse exister avant la vie ou que le monde puisse loger au sein d'une conscience. Il faut au contraire renvoyer dos-àdos toute interprétation idéaliste ou réaliste de l'existence puisque c'est seulement à travers la relation métabolique que connaissent le monde et l'existant ${ }^{20}$.

Ce dispositif rapidement évoqué jouera un rôle primordial dans Le principe responsabilité. Tout vivant métabolisant s'affirme en effet face au monde qu'il contribue à constituer, puisqu'en luttant contre la mort, il est conduit à ouvrir des possibilités et à revendiquer leur réalisation. Cette auto-affirmation qui exclut l'indifférence à soi fait que la vie est aussi valeur, c'est-à-dire qu'elle inclut dans son équipement ontologique l'exigence de réalisation de la possibilité qu'elle est ${ }^{21}$. Elle est même valeur en soi puisqu'en agissant de la sorte le vivant reconnaît que cette vie, qui exclut d'être sans poursuivre de fins, est bonne comme telle. Ajoutons, sans que Hans Jonas lui-même n'y insiste, qu'on peut s'assurer du caractère universel de cette valeur puisque tout vivant s'affirme selon ce mode et qu'on peut reconnaître dans les autres formes de vie semblable valorisation de soi. Indiquons également comment s'effectue le passage de l'axiologie à la déontologie : c'est parce que la technique humaine, pour la première fois, se révèle capable de démondanéiser la vie, y compris humaine, c'est-à-dire de la priver de son pouvoir de faire surgir des significations, que l'homme se découvre la responsabilité de la protéger en totalité. "Je peux donc je dois » signifie ici que de l'extension même de mon pouvoir procède un devoir. Il n'y a pas à interpréter ce «donc » en termes d'inférence logique puisqu'il s'agit d'une intuition ontologique, en vertu de laquelle, du fait même que je prends conscience de l'extension de mon pouvoir technique comme menaçant les possibilités de vie, surgit immédiatement un devoir correspondant qui m'interdit d'y porter atteinte de manière définitive ${ }^{22}$.

16 Ces brèves indications permettent déjà de comprendre que la vie abrite la valeur et que c'est d'elle que l'homme tire la sienne propre, valeur augmentée de ce qu'il porte un devoir de responsabilité envers la vie dans son ensemble du fait même de l'extension de son pouvoir et de sa «conscience ». En outre, en pensant la vie depuis son ouverture au monde, Jonas se donne les moyens de penser l'autonomie à partir d'un manque fondamental - celle du vivant qui résiste à la mort et ouvre ce faisant des horizons - en quoi consiste sa vulnérabilité. Il n'y a pas d'alternative entre ces deux notions et, puisque la liberté se pense à partir de cette fragilité, il n'y a pas lieu de détacher le respect de la personne humaine de son inscription corporelle, ce qui autorise une éthique médicale 
ayant pour fondement la vulnérabilité en tant qu'elle fait signe vers un horizon d'autonomie. Symétriquement, si tout vivant est de structure métabolique, alors son autonomie ne fait pas non plus alternative avec sa vulnérabilité. Sa capacité d'autorénovation présuppose le manque qu'il est: vulnérable en ce qu'il ne peut continuer d'exister qu'à condition de satisfaire de manière inlassable ses lacunes, autonome en ce qu'il jouit par lui-même du pouvoir de les satisfaire ${ }^{23}$. Puisque cette auto-affirmation est la manifestation d'une valeur, il faut conclure qu'un sujet apte à identifier et à agir en fonction de cette valeur éprouvera du respect pour la vie du vivant. À ce titre une éthique environnementale doit pouvoir être déduite de la structure même du raisonnement jonassien.

Pour autant, force est de remarquer que de telles implications sont compromises par la tendance même de Jonas à penser la structure métabolique du vivant de manière étroitement autocentrée et solitaire au détriment d'une conception relationnelle (qui par ailleurs permettra de renouveler le sens qu'il faut donner au couple autonomievulnérabilité). Ce manque s'exprime, à notre sens, aussi bien dans l'éthique médicale qu'il a mise en œuvre que dans l'éthique environnementale qu'il n'a pas développée. On pourrait dire en effet que cette dernière est impossible précisément parce que la valeur de la vie reste cantonnée à celle de l'individu ${ }^{24}$. Il est donc non seulement impossible au philosophe d'accorder une valeur à la terre mais également aux ensembles écosystémiques qui font fond sur un réseau de relations relativement unifiées entre différentes formes de vie.

Mais l'éthique médicale elle-même souffre d'une ambiguïté puisque tantôt Jonas insiste sur la préservation des générations futures, sur l'humanité prise en général dans son développement tempore ${ }^{25}$, tantôt sur le respect que l'on doit à la personne prise dans sa singularitée ${ }^{26}$. Ici encore cette ambiguïté trouve sa source dans un défaut de pensée de la relation. Faute de thématiser au plan de la biologie philosophique une relation des vivants entre eux, en particulier des vivants humains, au sein de laquelle l'horizon concret de l'humanité puisse se dessiner, Jonas est conduit à penser la responsabilité à l'égard des générations futures selon la catégorie de l'universalité abstraite. Certes l'humanité est comprise selon son sens temporel, donc comme ouverture vers l'avenir, mais elle ne l'est pas à partir des relations concrètes avec les individus du présent. L'opposition entre un souci abstrait pour les générations futures, qui ne concerne personne puisqu'elles ne sont pas encore là, et un souci, que rien ne justifie éthiquement, pour les individus particuliers doit être dépassée en comprenant comment le souci pour l'humanité dans son devenir temporel et éthique se joue dans la relation à tel ou tel autre et comment la relation à tel autre implique une dimension universelle qui dépasse sa particularité. C'est à cette condition que la responsabilité pour l'universel pourra s'incarner dans des relations particulières et que le particulier pourra s'épaissir du poids d'une responsabilité universelle. De ce dernier point de vue, seule une description de la relation à l'homme vivant souffrant, en tant qu'en elle se fait jour un devoir de responsabilité envers l'humanité prise en un sens temporel, si bien que cette responsabilité doit nécessairement se traduire sous la forme de responsabilités singulières, permettrait de lever cette ambiguïté. C'est pourquoi nous souhaiterions à présent insister sur la manière dont nous pouvons interpréter, voire dépasser, l'éthique jonassienne de manière à penser les principes et les attendus non seulement d'une éthique médicale et d'une éthique environnementale mais également de leur compatibilité au sein d'un système unifié de l'éthique. 


\section{Vers une conception relationnelle de l'éthique de la vie}

19 C'est en effet à partir d'une éthique de la vie relationnelle que nous pourrons penser l'unité et la cohérence de ses champs d'application médicale et environnementale. L'éthique jonassienne se propose en effet de justifier un principe formel commandant de prendre en compte les conséquences de notre agir technologique à la fois sur les écosystèmes et sur l'humanité comprise en son « socle » biologique. Un tel impératif qui nous enjoint à la responsabilité n'a de sens qu'à condition de reconnaître au préalable, comme nous l'avons $\mathrm{vu}$, une valeur en soi à la nature et à la vie humaine. C'est en effet à condition de les considérer comme ayant une valeur absolue qu'on peut tenir pour une faute le fait de leur porter atteinte par l'insouciance de notre agir, la déontologie supposant une axiologie. Et voilà pourquoi nous devons anticiper la portée de notre pouvoir et évaluer, autant que possible, le sens des transformations technologiques.

Il serait alors presque aussi préjudiciable de vouloir séparer la responsabilité que nous devons montrer envers la nature et celle que nous devons adresser à l'homme que de séparer celle que nous devons à l'humanité de l'homme et à son existence biologique, compte-tenu dans chacun de ces deux cas du caractère relationnel qui lie un aspect à l'autre. En d'autres termes et au plan axiologique, c'est parce que je fais l'expérience, d'une part, de la valeur pour mon humanité de ma condition incarnée et, d'autre part, de la valeur de mon inscription dans une nature, que je peux en déduire l'existence de valeurs relationnelles multiples au sein de la nature. La vie de mon humanité s'atteste aussi bien dans sa relation à un environnement que dans une relation à soi-même par le biais de mon corps, je peux en éprouver la valeur en ce que cette expérience est également l'épreuve d'une affirmation et d'un maintien positif de la dynamique de cette relation, ce que l'on peut aussi appeler santé, terme qui n'a pas qu'un sens descriptif mais également une dimension axiologique puisqu'elle est un bien. Au lieu donc d'en rester à la conception individuo-centrée du vivant propre à Jonas, commençons d'indiquer en quel sens une relecture relationnelle de sa biologie permettrait de reconfigurer son éthique. Évoquons alors les deux ordres d'expérience mentionnés plus haut: la valeur du lien de l'homme à l'environnement et à lui-même grâce à son corps.

21 De fait l'homme s'inscrit au sein d'écosystèmes qu'il contribue à dessiner tandis que ces écosystèmes contribuent également à son devenir. Uexküll, par exemple, montre en quel sens on peut identifier des mondes animaux en sus du monde humain ${ }^{27}$. La nature est enchevêtrement de mondes ${ }^{28}$. On pourra certes faire valoir qu'il y a une différence entre ces « centres » de valeur que sont les vivants, hommes compris, et la nature, qui, quant à elle, inclut aussi le sol, le ciel ou les océans. Pourtant, ce que nous distinguons là comme étant des réalités matérielles se trouve en vérité être des lieux de vie. Ils ne doivent ce qu'ils sont qu'à l'intervention des "sujets naturels" qui en retour se constituent seulement à condition de prendre en compte cela qui deviendra leur environnement. Un sol, par exemple, n'est donc jamais seulement un ensemble de particules analysable d'après les lois de la physico-chimie ${ }^{29}$. C'est une terre dont la composition se modifie grâce aux espèces auxquelles elle permet de vivre. Il faut donc insister sur la solidarité de destin entre la nature (mondes animaux et végétaux qui impliquent une appropriation des lieux de vie matériels) et l'homme, sur la valeur de ce lien puisqu'il permet à chacun des termes en jeu de s'affirmer en tant que vivant. 

humanité s'incarne. Peut-on modifier les conditions de la relation qu'il noue à sa naissance et à sa mortalité sans lui faire perdre l'autonomie et la capacité de responsabilité qui sont siennes? Lorsque Miroslav Radman dresse les perspectives transhumaines d'une génétique affranchie de la nécessité de mourir ${ }^{30}$, ne faut-il pas se tourner du côté de José Saramago pour s'interroger sur les conséquences lourdes d'inhumanité qu'une " grève de la mort » risquerait d'entraner ${ }^{31}$ ? Lorsque le clonage nous est présenté comme remède aux hasards inquiétants d'une naissance parfois peu avare de mauvaises surprises, ne faut-il pas questionner ce que serait une vie sans « la liberté de se découvrir comme une surprise pour soi-même et pour les autres $\|^{32}$ ? pouvoir être complétée par une déontologie, c'est dans la mesure où la santé, qui est un terme relationnel, de l'homme et de la nature n'est pas simplement un terme descriptif neutre. Elle inclut immédiatement la reconnaissance d'un certain bien : le soin, entretien ou secours d'urgence, est un devoir dès lors qu'il s'adresse à un type de vivant capable de le reconnaître et de l'accomplir si bien qu'on peut imputer à celui qui refuse de l'accorder le qualificatif d'irresponsable.

Cette responsabilité de l'homme envers le lien qui unit son humanité et sa vie empirique, comprise d'une part comme rapport à l'environnement, d'autre part comme rapport à son corps propre conduit à rapprocher l'éthique environnementale et l'éthique médicale. On doit certes distinguer avec force une médecine qui prend pour objet le corps vivant et l'écologie qui s'intéresse à des écosystèmes. Pour autant, cette séparation, essentielle au plan méthodologique et nécessaire à la constitution de ces disciplines, doit être surmontée au plan compréhensif qui est celui de la philosophie. Certes l'analogie entre les deux systèmes permet d'identifier des ressemblances : l'un et l'autre manifestent une unité irréductible à une explication mécaniste ou finaliste, une identité irréductible à celle de la substance matérielle ou spirituelle, une autonomie dans la vulnérabilité qui appelle à un « prendre soin », et semblent viser une certaine perpétuation dynamique de « soi » par le renouvellement interne de leurs composantes. Certes cette analogie permet également de relever des différences : la solidarité intra-organique est plus « forte » que l'échange entre organismes, la dimension subjective du corps propre est éloignée de l'éventuelle «subjectivité " que l'on pourrait accorder à l'écosystème ${ }^{33}$, etc. Il faut néanmoins reconnaître, par-delà une telle analogie, des interférences réelles entre la vie humaine et la vie terrestre. Un écosystème dégradé n'est pas sans influence sur la santé humaine. La surpopulation, que renforcerait la perspective d'une immortalité biologique, peut être préjudiciable aussi bien à la nature qu'à l'homme. Soigner un corps humain n'exclut donc pas de prendre en compte l'environnement dans lequel il se trouve, et la santé d'un écosystème n'est pas indemne de la qualité de l'activité humaine qui s'exerce en son sein. C'est donc dans l'être même que se dessine une interdépendance, et une forme de communauté de destin entre la nature et la vie humaine. Se reconnaître en charge de l'équilibre du lien entre l'activité humaine et le système de la nature n'est rien d'autre que mettre en œuvre le principe responsabilité. Mais c'est précisément cette dimension déontologique et non pas simplement axiologique de l'éthique sur laquelle il faut insister à présent. Car il ne suffit pas d'affirmer la valeur en soi de la relation vitale qui relie l'homme à lui-même et à l'environnement pour affirmer l'existence d'un devoir. L'axiologie est sans doute la condition nécessaire d'une déontologie mais non sa condition suffisante. Insistons donc sur la justification d'un devoir de responsabilité à l'égard de la 
vie comprise comme relation, en tant qu'elle permet de conjoindre ce que l'humanisme abstrait séparait : l'autonomie et la vulnérabilité. Le passage de la structure de la valeur à celle d'un devoir, qui commande de répondre des conséquences de son agir, exige de rendre raison du sujet en tant qu'il est capable d'identifier la valeur et de la respecter. Or, si le sujet éthique peut se reconnaître des devoirs, c'est qu'il fait en lui-même l'expérience d'une autonomie dans la vulnérabilité qui, d'une part, non seulement le rend réceptif à la vulnérabilité d'autrui mais également lui permet d'en identifier le vœu d'autonomie, et d'autre part, lui permet d'intervenir activement (en vertu de son autonomie) mais aussi précautionneusement (en vertu de sa vulnérabilité) afin d'exercer sa responsabilité. Puisque la vie est, comme nous l'avons vu, ce type d'être relationnel dont l'autonomie présuppose la reconnaissance à des niveaux divers de sa vulnérabilité (c'est-à-dire de sa dépendance aux autres vivants), la responsabilité sera donc pour l'homme la reconnaissance d'une obligation de soin non pas tant à l'égard de la vie du malade et de l'écosystème, comme s'ils étaient des entités closes, qu'à l'égard du vivre, c'est-à-dire de la relation qu'il nourrit à eux, en vue de préserver leur autonomie par la prise en charge de leur vulnérabilité. Insistons ici sur trois dimensions de la responsabilité : médicale, environnementale et « humaniste ».

26 Jonas cherchant à justifier le principe responsabilité prend comme paradigme la responsabilité parentale. La précarité d'un nourrisson laissé à lui-même nous intime le devoir de le prendre en charge. Mais il ne faut pas s'y tromper. Cette prise en charge n'est valable qu'en vertu de l'horizon d'autonomie qui habite le petit d'homme et qu'il s'agit de faire croître. C'est parce qu'il est promesse de liberté, cette promesse étant elle-même la manifestation d'une liberté à l'œuvre dans la vie ${ }^{34}$, et c'est pour porter cette promesse à sa destination que le parent est responsable de l'enfant. C'est également la valeur de cette autonomie qui limite l'office de la tutelle au temps que la nature lui assigne pour exercer sa responsabilité. Non seulement donc l'être vulnérable conserve un rapport à la valeur d'autonomie mais en outre le responsable, qui est autonome, ne peut concevoir le sens de sa mission que parce qu'il a été vulnérable et sans doute aussi parce qu'il le demeure, bien que dans une moindre mesure que l'enfant dont il a la charge. Le parent a lui-même été enfant et ne doit ce qu'il est qu'aux soins qu'il a reçu. En outre, il ne peut exercer sa responsabilité actuelle que sous la condition d'un réseau de solidarité qui est vital au soin de l'enfant (la famille, les amis, l'institution médicale, etc.). On peut alors appliquer un tel modèle à la figure du malade et aux existences fragilisées et inspirer ainsi une éthique médicale de la responsabilité.

Symétriquement, le devoir de responsabilité a pour but de maintenir une certaine autonomie des écosystèmes. Il s'agit de prendre acte de leurs fragilités pour leur assurer les conditions dont ils ont besoin afin de rendre possible le renouvellement de l'unité qu'ils sont. S'il est question de préservation de la nature, celle-ci ne doit donc pas être conçue au sens d'une conservation statique - comme ce pourrait être le cas d'une réserve naturelle pure de toute introduction d'espèce extérieure - mais d'un entretien dynamique. À la patrimonalisation mortifère à laquelle correspond une mise sous tutelle qui dénie à l'écosystème la capacité d'évoluer, il faut préférer une préservation des logiques dynamiques qui assurent la vie, et même la santé, d'un écosystème en quoi consiste l'autonomie dont il est capable ${ }^{35}$. Mais c'est dans la conscience que notre autonomie suppose la reconnaissance de notre vulnérabilité à l'environnement, que se trouve la clef de notre sens des responsabilités, puisque c'est parce que nous sommes 
autonomes que nous pouvons intervenir et parce que nous sommes vulnérables que la vulnérabilité de la nature trouve un écho en nous.

Pour autant ces responsabilités envers le malade et la nature supposent la référence aux générations futures, à l'être de l'homme compris dans sa dimension temporelle. C'est en effet parce qu'il n'y a d'humanité authentique que capable de responsabilité qu'il faut élever l'enfant dans l'horizon d'un tel fardeau, qu'il faut guérir le malade mais aussi qu'il faut protéger la nature. Le principe responsabilité préserve donc une orientation humaniste puisqu'il s'agit de prendre l'homme comme objet privilégié de la responsabilité, mais cela dans la mesure stricte où c'est le sujet par excellence de la responsabilité, celui dont il faut préserver la compétence éthique, celui dont l'autonomie ancrée dans une vulnérabilité d'essence biologique lui commande d'assurer la perpétuation de cette autonomie vulnérable. L'homme d'aujourd'hui se reconnait une responsabilité envers l'humanité de demain parce que cette dernière est fragile et que son autonomie à venir dépend des générations actuelles. Mais si les hommes d'aujourd'hui doivent exercer cette responsabilité, c'est aussi parce qu'ils jouissent d'une autonomie conditionnée par le legs éthique des générations passées, donc par la reconnaissance de leur propre vulnérabilité au regard des hommes du passé ${ }^{36}$. C'est à condition de mettre l'accent sur cette dimension relationnelle des générations entre elles, dimension qui a son origine dans le vivre, c'est-à-dire dans la part empirique de l'homme en tant qu'elle le porte à se reconnaître responsable des écosystèmes et des existences fragiles, qu'on peut penser l'unité de l'éthique médicale et environnementale et qu'on peut comprendre comment la responsabilité envers les générations futures cesse d'être un principe abstrait au profit d'une responsabilité concrète, telles qu'elles s'incarnent dans les relations parentales, médicales, environnementales.

\section{NOTES}

2. Voir par ex. C. et. R. Larrère, Du bon usage de la nature (1997), Paris, Champs essais, 2009 ; C. Pelluchon, L'autonomie brisée, Paris, PUF, 2009 ; S. Laugier, Tous vulnérables ?, Paris, Payot, 2012.

3. Voir par ex. P. Descamps, Le sacre de l'espèce humaine, Paris, PUF, 2009 ; M. Foessel, « Kant ou les vertus de l'autonomie ", Études, mars 2011 ; M. Heidegger, "Le Tournant », Questions IV, trad. J. Lauxerois et C. Roëls, Paris, Gallimard, 1990, p. 319.

4. Nous serons amenés à questionner l'opposition entre un fait apparemment axiologiquement neutre - la vulnérabilité - qui appelle pourtant une réponse morale et une valeur apparemment trans-empirique - l'autonomie comprise comme obéissance à la loi morale - qui se déduit pourtant d'un fait de la raison ( = la loi morale).

5. Discussion et Responsabilité, II. Contribution à une éthique de la responsabilité, trad. C. Bouchindhomme et R. Rochlitz, Paris, Le Cerf, 1998, pp. 9-38.

6. «Technology, Medicine and Ethics » in Hans Jonas, Graduate Faculty Philosophy Journal 23(2): 155-182 (2002). Cf. également L. Benaroyo, Éthique et responsabilité en médecine, Genève, éd. Médecine \& hygiène, 2006, p. 51. 
7. Le principe responsabilité (1990), trad. J. Greisch, Paris, Champs Flammarion, 1998, pp. 253-254. Noté PR. Cf. également la critique de P. Ricœur, «La responsabilité et la fragilité de la vie », Le messager européen 5, Gallimard, 1991.

8. Ici comme là, l'autonomie s'oppose donc à la vulnérabilité. Le sens de l'une et de l'autre est irréductible à la possibilité d'une synthèse, bien que l'éthique fasse nécessairement fond sur les deux. Or ne faudrait-il pas considérer, comme nous souhaitons le montrer et sous peine d'être conduit à des difficultés dirimantes, que l'autonomie est une valeur précisément parce qu'elle consiste en un dépassement permanent de la vulnérabilité à laquelle elle reste ontologiquement liée?

9. «La nature a-t-elle une valeur en elle-même?», Éthique de la Terre [ET], Paris, éd. B. Lanaspeze, Wildproject, p. 121.

10. ET, pp. 49 sqq.

11. ET, pp. 80-81.

12. ET, p. 84 .

13. Bergson, sans d'abord considérer le phénomène moral, mettra en lumière le hiatus entre l'explication darwinienne du vivant et la compréhension véritable de la vie comme dynamique créatrice. Il y a plus dans la vie qu'un mécanisme de type darwinien. Ainsi, dans le premier chapitre de l'Évolution créatrice, il montre en quel sens les principes darwiniens ne permettent pas de comprendre la créativité à l'œuvre dans la vie et, dans les Deux sources de la morale et de la religion, ce que le fait de la morale nous oblige à conclure à propos de l'ontologie de la vie. L'éthique de la vie requiert une ontologie irréductible à l'explication, en partie mécaniste, de Darwin. Certes Callicott fait valoir que la nature ne peut être amorale puisqu'elle produit un être moral (ET, p. 81). Mais le principe de continuité ainsi invoqué n'est pas congruent avec l'ontologie de la nature d'inspiration darwinienne revendiquée par Callicott.

14. Accrétion (par ex. ET, p. 161).

15. ET, p. 164.

16. Pour préciser l'exemple, cf. ET, pp. 165-166.

17. Paris, PUF, 1997. Noté PE.

18. PE, p. 123.

19. Trad. D. Lories, Bruxelles, De Boeck, 2001.

20. Sur tous ces points ici simplement évoqués, nous nous permettons de renvoyer à E. Pommier, Ontologie de la vie et éthique de la responsabilité selon Hans Jonas, chap. 1, Paris, Vrin, 2013.

21. PR, p. 157.

22. PR, pp. 248-249.

23. C'est d'ailleurs une telle considération ontologique que Jonas estime être à l'arrière-plan de l'éthique kantienne du respect de la personne, cf. PR, p. 177.

24. Cf. J.-C. Gens, «L'assise ontologique et les arrière-plans cosmogoniques de l'éthique jonassienne ", in L'éthique de la vie chez Hans Jonas, C. Larrère et E. Pommier, Paris, Publications de la Sorbonne, 1993, p. 157.

25. Cf. par exemple Hans Jonas, L'art médical et la responsabilité humaine, trad. E. Pommier, préface d'E. Hirsch, Paris, Le Cerf, 2012, pp. 63-76. Noté AM.

26. AM, pp. 47 sqq.

27. Mondes animaux et monde humain, trad. P. Muller, Paris, Denoël, 1965.

28. Id. p. 89.

29. J.M.G. Le Clezio, Ourania, Paris, Folio, 2008, pp. 87-98.

30. M. Radman avec D. Carton, Au-delà de nos limites biologiques, Paris, Plon, 2011.

31. J. Saramago, Les intermittences de la mort, trad. G. Leidrich, Paris, Points Seuil, 2008. On doit également se tourner du côté de "Fardeau et bénédiction de la mortalité ", Évolution et Liberté, trad. S. Cornille et P. Ivernel, Paris, Payot Rivages, 2000, dans lequel Jonas montre les 
conséquences d'une disparition de la mortalité tant au plan de la construction individuelle qu'au plan générique.

32. AM, p. 73.

33. A. Leopold, Almanach d'un comté des sables, trad. A. Gibson, Paris, GF, 2000, p. 168.

34. La liberté est en effet le phénomène de la vie puisque l'autonomie du métabolisme consiste dans ce pouvoir de renouveler ses composantes matérielles.

35. Ainsi l'introduction d'espèces étrangères ne doit pas être écartée a priori.

36. Il est vrai que ceux-ci n'étaient pas encore en mesure de mettre en danger, à cause de leur pouvoir technique, l'autonomie de l'humanité. 